\title{
Catalytic, Asymmetric trans-Selective Hetero Diels-Alder Reactions Using a Novel Chiral Zirconium Catalyst
}

\author{
Yasuhiro Yamashita, Susumu Saito, Haruro Ishitani, and Shū Kobayashi* \\ Graduate School of Pharmaceutical Sciences, The University of Tokyo, CREST, \\ Japan Science and Technology Corporation (JST), \\ Hongo, Bunkyo-ku, Tokyo 113-0033 Japan
}

\section{Supporting Information}

\section{Experimental Details}

\section{General}

Melting points were uncorrected. ${ }^{1} \mathrm{H}$ and ${ }^{13} \mathrm{C}$ NMR spectra were recorded on a JEOL JNM-LA300, JNM-LA400, or JNM-LA500 spectrometer in $\mathrm{CDCl}_{3}$ unless otherwise noted. Tetramethylsilane (TMS) served as internal standard $(\delta=0)$ for ${ }^{1} \mathrm{H}$ $\mathrm{NMR}$, and $\mathrm{CDCl}_{3}$ was used as internal standard $(\delta=77.0)$ for ${ }^{13} \mathrm{C}$ NMR. IR spectra were measured with JASCO FT/IR-610 spectrometers. Optical rotations were measured with a JASCO P-1010 polarimeter. High-performance liquid chromatography was carried out using following apparatuses; SHIMADZU LC-10AT (liquid chromatograph), SHIMADZU SPD-10A (UV detector), and SHIMADZU CR6A Chromatopac. Mass spectrometry analysis was carried out using SHIMADZU GCMS-QP5050A. Column chromatography was conducted on Silica gel 60 (Merck) and preparative thin-layer chromatography was carried out using Wakogel B-5F. Toluene was distilled and dried over MS 4A. $\mathrm{Zr}\left(\mathrm{O}^{t} \mathrm{Bu}\right)_{4}$ was purchased from Trichemical Laboratory Co., Ltd.. Employed aldehydes were purchased from Tokyo Kasei Kogyo Co., Ltd. Danishefsky's dienes were prepared according to the 
literature. ${ }^{1}$ Propanol was distilled in the presence of magnesium propoxide. All reactions were carried out under argon atmosphere in dried glassware.

Typical experimental procedure for asymmetric hetero Diels-Alder reactions using a chiral zirconium catalyst prepared from $\operatorname{Zr}\left(\mathrm{O}^{t} \mathrm{Bu}\right)_{4}$ and $(R)-$

3,3'-I2BINOL: A typical experimental procedure is described for the reaction of benzaldehyde with Danishefsky's diene 1b. To a suspension of $(R)-3,3$ '-diiodo-1,1'binaphthalene-2,2'-diol ((R)-3,3'- $\left.\mathrm{I}_{2} \mathrm{BINOL}, 0.048 \mathrm{mmol}\right)$ in toluene $(0.5 \mathrm{~mL})$, was added $\mathrm{Zr}\left(\mathrm{O}^{t} \mathrm{Bu}\right)_{4}(0.040 \mathrm{mmol})$ in toluene $(0.5 \mathrm{~mL})$ at room temperature, and the solution was stirred for $30 \mathrm{~min}$. Then propanol $(0.32 \mathrm{mmol})$ and $\mathrm{H}_{2} \mathrm{O}(0.080 \mathrm{mmol})$ in toluene $(0.3 \mathrm{~mL})$ were added and the whole was stirred for $3 \mathrm{~h}$ at room temperature. After cooling at $-78{ }^{\circ} \mathrm{C}$, benzaldehyde $(0.40 \mathrm{mmol})$ in toluene $(0.35 \mathrm{~mL})$ and diene $\mathbf{1 b}(0.48 \mathrm{mmol})$ in toluene $(0.35 \mathrm{~mL})$ were successively added. The mixture was warmed to $-20{ }^{\circ} \mathrm{C}$ and stirred for $18 \mathrm{~h}$, then saturated aqueous $\mathrm{NaHCO}_{3}$ $(10 \mathrm{ml})$ was added to quench the reaction. After added dichloromethane $(10 \mathrm{~mL})$, the organic layer was separated and the aqueous layer was extracted twice with dichloromethane $(10 \mathrm{~mL} \times 2)$. The organic layers were combined and dried over anhydrous $\mathrm{Na}_{2} \mathrm{SO}_{4}$. After filtration and concentration under reduced pressure, the residue was treated with TFA $(0.5 \mathrm{~mL})$ in dichloromethane $(8 \mathrm{~mL})$ for $1 \mathrm{~h}$ at $0{ }^{\circ} \mathrm{C}$. After the solution was basicified with saturated aqueous $\mathrm{NaHCO}_{3}(20 \mathrm{ml})$, the organic layer was separated and extracted with dichloromethane $(10 \mathrm{~mL}$ x 2). The organic layers were combined and dried over anhydrous $\mathrm{Na}_{2} \mathrm{SO}_{4}$. After filtration and concentration under reduced pressure, the crude product was purified by preparative thin layer chromatography (benzene-ethyl acetate=20:1) to afford the desired product. The optical purity was determined by HPLC analysis using a chiral column (see following analytical data).

Typical experimental procedure for asymmetric hetero Diels-Alder reactions using a chiral zirconium catalyst prepared from $\mathrm{Zr}\left(\mathrm{O}^{t} \mathrm{Bu}\right)_{4}$ and $(R)$ 3,3'-I $\mathbf{I}_{2}-6,6^{\prime}-\mathbf{X}_{2} \mathbf{B I N O L}\left(\mathbf{X}=\mathbf{I}, \mathbf{C}_{2} \mathbf{F}_{5}\right)$ : A typical experimental procedure is described for the reaction of benzaldehyde with Danishefsky's diene 1d. To a suspension of 
(R)-3,3'-diiodo-6,6' -disubstituted-1,1'-binaphthalene-2,2' -diol ((R)-3,3'- $\mathrm{I}_{2}-6,6$ ' $\left.\mathrm{X}_{2} \mathrm{BINOL}\left(\mathrm{X}: \mathrm{C}_{2} \mathrm{~F}_{5}, \mathrm{I}\right), 0.060 \mathrm{mmol}\right)$ in toluene $(0.5 \mathrm{~mL})$, was added $\mathrm{Zr}\left(\mathrm{O}^{t} \mathrm{Bu}\right)_{4}$ $(0.040 \mathrm{mmol})$ in toluene $(0.5 \mathrm{~mL})$ at room temperature, and the solution was stirred for $3 \mathrm{~h}$. Then propanol $(0.32 \mathrm{mmol})$ and $\mathrm{H}_{2} \mathrm{O}(0.080 \mathrm{mmol})$ in toluene $(0.3 \mathrm{~mL})$ were added and the whole was stirred for $30 \mathrm{~min}$ at room temperature. After cooling at -78 ${ }^{\circ} \mathrm{C}$, benzaldehyde $(0.40 \mathrm{mmol})$ in toluene $(0.35 \mathrm{~mL})$ and diene $1 \mathbf{d}(0.48 \mathrm{mmol})$ in toluene $(0.35 \mathrm{~mL})$ were successively added. The mixture was warmed to $-20{ }^{\circ} \mathrm{C}$ and stirred for $18 \mathrm{~h}$, then saturated aqueous $\mathrm{NaHCO}_{3}(10 \mathrm{~mL})$ was added to quench the reaction. After added dichloromethane $(10 \mathrm{~mL})$, the organic layer was separated and the aqueous layer was extracted twice with dichloromethane $(10 \mathrm{~mL} \times 2)$. The organic layers were combined and dried over anhydrous $\mathrm{Na}_{2} \mathrm{SO}_{4}$. After filtration and concentration under reduced pressure, the residue was treated with TFA $(0.5 \mathrm{~mL})$ in dichloromethane $(8 \mathrm{~mL})$ for $1 \mathrm{~h}$ at $0{ }^{\circ} \mathrm{C}$. In case of the reaction of $\alpha, \beta$-unsaturated aldehyde, $\mathrm{Sc}(\mathrm{OTf})_{3}(0.040 \mathrm{mmol}, 10 \mathrm{~mol} \%$ to employed aldehyde) was used instead of TFA in dichloromethane at room temperature for $12 \mathrm{~h}$. After the solution was basified with saturated aqueous $\mathrm{NaHCO}_{3}(20 \mathrm{~mL})$ the organic layer was separated and extracted with dichloromethane $(10 \mathrm{~mL}$ x 2$)$. The organic layers were combined and dried over anhydrous $\mathrm{Na}_{2} \mathrm{SO}_{4}$. After filtration and concentration under reduced pressure, the desired trans and cis diastereomers were separated and purified by preparative thin layer chromatography (benzene-ethyl acetate=20:1). The optical purity was determined by HPLC analysis using a chiral column (see below).

(R)-2-Phenyl-2,3-dihydro-4H-pyran-4-one: ${ }^{2}[\alpha]_{\mathrm{D}}{ }^{26}=-97.1\left(\right.$ c $\left.0.69, \mathrm{CHCl}_{3}\right)$ (97\% ee). lit. $)^{2}[\alpha]_{\mathrm{D}^{23}}=+88.4\left(\mathrm{c} 0.529, \mathrm{CHCl}_{3}\right)(87 \%$ ee, $S$-form); IR [cm-1] 3063, 2902, 1680, 1595, 1496, 1455, 1403, 1270, 1227, 1209; ${ }^{1} \mathrm{H}$ NMR $\left(\mathrm{CDCl}_{3}\right) \delta 2.66(\mathrm{dd}$, $1 \mathrm{H}, J=17,3.5 \mathrm{~Hz}), 2.90(\mathrm{dd}, 1 \mathrm{H}, J=17,14 \mathrm{~Hz}), 5.42(\mathrm{dd}, 1 \mathrm{H}, J=15,3.7 \mathrm{~Hz}), 5.52$ $(\mathrm{d}, 1 \mathrm{H}, J=6.0 \mathrm{~Hz}), 7.3-7.4(\mathrm{~m}, 5 \mathrm{H}), 7.48(\mathrm{~d}, 1 \mathrm{H}, J=6.0 \mathrm{~Hz}) ;{ }^{13} \mathrm{C} \mathrm{NMR}\left(\mathrm{CDCl}_{3}\right) \delta$ 43.3, 81.0, 107.3, 126.0, 128.8, 128.9, 137.8, 163.1, 192.1; HPLC, Daicel Chiralcel $\mathrm{OD}$, hexane $/{ }^{i} \mathrm{PrOH}=40 / 1$, flow rate $=1.0 \mathrm{~mL} / \mathrm{min}: t_{\mathrm{R}}=25.1 \mathrm{~min}(S), t_{\mathrm{R}}=30.1 \mathrm{~min}$ $(R)$. 
2-(p-Chlorophenyl)-2,3-dihydro-4H-pyran-4-one: ${ }^{2}[\alpha]_{\mathrm{D}}{ }^{19}=-62.4$ (c 0.56, $\mathrm{CHCl}_{3}$ ) (84\% ee); IR [cm-1] 3063, 2906, 1907, 1675, 1599, 1494, 1402, 1267, 1227 , $1208 ;{ }^{1} \mathrm{H} \mathrm{NMR}\left(\mathrm{CDCl}_{3}\right) \delta 2.64(\mathrm{dd}, 1 \mathrm{H}, J=17,3.7 \mathrm{~Hz}), 2.85(\mathrm{dd}, 1 \mathrm{H}, J=17,14 \mathrm{~Hz})$, $5.41(\mathrm{dd}, 1 \mathrm{H}, J=14,3.5 \mathrm{~Hz}), 5.53(\mathrm{~d}, 1 \mathrm{H}, J=6.1 \mathrm{~Hz}), 7.34(\mathrm{~d}, 2 \mathrm{H}, J=8.7 \mathrm{~Hz}), 7.39$ (d, 2H, 8.7 Hz), $7.47(\mathrm{~d}, 1 \mathrm{H}, J=6.1 \mathrm{~Hz}) ;{ }^{13} \mathrm{C} \mathrm{NMR}\left(\mathrm{CDCl}_{3}\right) \delta 43.3,80.2,107.5$, 127.4, 129.0, 134.7, 136.3, 162.9, 191.6; HPLC, Daicel Chiralcel OD, hexane/ ${ }^{i} \mathrm{PrOH}$ $=40 / 1$, flow rate $=1.0 \mathrm{~mL} / \mathrm{min}: t_{\mathrm{R}}=27.2 \mathrm{~min}$ (minor), $t_{\mathrm{R}}=35.9 \mathrm{~min}$ (major).

(S)-2-Phenethyl-2,3-dihydro-4H-pyran-4-one: ${ }^{2}[\alpha]_{\mathrm{D}}^{19}=-93.4$ (c 1.34, $\mathrm{CHCl}_{3}$ ) (85\% ee); IR [cm-1] 3023, 2931, 2632, 1674, 1599, 1495, 1455, 1402, 1274. ${ }^{1} \mathrm{H} \mathrm{NMR}\left(\mathrm{CDCl}_{3}\right) \delta 1.95(\mathrm{~m}, 1 \mathrm{H}), 2.15(\mathrm{~m}, 1 \mathrm{H}), 2.42(\mathrm{dd}, 1 \mathrm{H}, J=17,3.8 \mathrm{~Hz}), 2.55$ $(\mathrm{dd}, 1 \mathrm{H}, J=17,13 \mathrm{~Hz}), 2.80(\mathrm{~m}, 2 \mathrm{H}), 4.39(\mathrm{~m}, 1 \mathrm{H}), 5.40(\mathrm{~d}, 1 \mathrm{H}, J=6.0 \mathrm{~Hz}), 7.1-7.3$ $(\mathrm{m}, 5 \mathrm{H}), 7.38(\mathrm{~d}, 1 \mathrm{H}, J=6.1 \mathrm{~Hz}) ;{ }^{13} \mathrm{C} \mathrm{NMR}\left(\mathrm{CDCl}_{3}\right) \delta 30.9,36.0,41.8,78.4,107.1$, 126.2, 128.4, 128.6, 140.6, 163.1, 192.4; HPLC, Daicel Chiralcel OD, hexane/ $i \mathrm{PrOH}$ $=9 / 1$, flow rate $=1.0 \mathrm{~mL} / \mathrm{min}: t_{\mathrm{R}}=21.7 \min (R), t_{\mathrm{R}}=43.8 \mathrm{~min}(S)$.

2-(p-Tolyl)-2,3-dihydro-4H-pyran-4-one: $[\alpha]_{\mathrm{D}}{ }^{19}=-27.5\left(\right.$ c $\left.0.26, \mathrm{CHCl}_{3}\right)$ (92\% ee); IR $\left[\mathrm{cm}^{-1}\right] 2921,1672,1596,1515,1405,1271,1220 ;{ }^{1} \mathrm{H} \mathrm{NMR}\left(\mathrm{CDCl}_{3}\right) \delta$ $2.28(\mathrm{~s}, 3 \mathrm{H}), 2.53(\mathrm{dd}, 1 \mathrm{H}, J=17,3.5 \mathrm{~Hz}), 2.81(\mathrm{dd}, 1 \mathrm{H}, J=17,15 \mathrm{~Hz}), 5.29(\mathrm{dd}, 1 \mathrm{H}$, $J=14,3.3 \mathrm{~Hz}), 5.43(\mathrm{dd}, 1 \mathrm{H}, J=6.1,1.3 \mathrm{~Hz}), 7.1-7.3(\mathrm{~m}, 4 \mathrm{H}), 7.37(\mathrm{~d}, 1 \mathrm{H}, J=6.1$ $\mathrm{Hz}) ;{ }^{13} \mathrm{C} \mathrm{NMR}\left(\mathrm{CDCl}_{3}\right) \delta 21.2,43.3,81.0,107.3,126.1,129.5,134.8,138.9,163.2$, 192.3; HPLC, Daicel Chiralcel OD, hexane $/{ }^{i} \mathrm{PrOH}=40 / 1$, flow rate $=1.0 \mathrm{~mL} / \mathrm{min}: t_{\mathrm{R}}$ $=22.2 \min \left(\right.$ minor), $t_{\mathrm{R}}=27.1 \mathrm{~min}$ (major); HRMS $(\mathrm{m} / \mathrm{z})$ calcd. for $\mathrm{C}_{12} \mathrm{H}_{12} \mathrm{O}_{2}\left(\mathrm{M}^{+}\right)$: 188.0837; found: 188.0832 .

2-Pentyl-2,3-dihydro-4H-pyran-4-one: $[\alpha]_{\mathrm{D}}{ }^{19}=-131.4\left(\mathrm{c} 0.54, \mathrm{CHCl}_{3}\right)(83 \%$ ee); IR $\left[\mathrm{cm}^{-1}\right]$ 2935, 2865, 1678, 1599, 1466, 1403, 1274; ${ }^{1} \mathrm{H} \mathrm{NMR}\left(\mathrm{CDCl}_{3}\right) \delta 0.90$ $(\mathrm{t}, 3 \mathrm{H}, J=6.8 \mathrm{~Hz}), 1.3-1.5(\mathrm{~m}, 6 \mathrm{H}), 1.66(\mathrm{~m}, 1 \mathrm{H}), 1.81(\mathrm{~m}, 1 \mathrm{H}), 2.4-2.5(\mathrm{~m}, 1 \mathrm{H})$, $4.4(\mathrm{~m}, 1 \mathrm{H}), 5.4(\mathrm{dd}, 4 \mathrm{H}, J=5.9,0.7 \mathrm{~Hz}), 7.35(\mathrm{~d}, 1 \mathrm{H}, J=6.1 \mathrm{~Hz}) ;{ }^{13} \mathrm{C} \mathrm{NMR}$ $\left(\mathrm{CDCl}_{3}\right) \delta 13.9,22.4,24.4,31.4,34.3,41.8,79.5,106.9,163.3,192.7$; HPLC, Daicel Chiralcel OD, hexane $/ \mathrm{PrOH}=40 / 1$, flow rate $=0.5 \mathrm{~mL} / \mathrm{min}: t_{\mathrm{R}}=19.0 \mathrm{~min}($ minor $)$, 
$t_{\mathrm{R}}=21.1 \mathrm{~min}$ (major); HRMS $(\mathrm{m} / \mathrm{z})$ calcd. for $\mathrm{C}_{10} \mathrm{H}_{16} \mathrm{O}_{2}\left(\mathrm{M}^{+}\right)$: 168.1150; found: 168.1132 .0

3,5-Dimethyl-2-phenyl-2,3-dihydro-4H-pyran-4-one: trans isomer: $[\alpha]_{\mathrm{D}}{ }^{26}=$ -35.1 (c 0.77, $\left.\mathrm{CHCl}_{3}\right)(96 \%$ ee); IR [cm-1] 3037, 2926, 1964, 1725, 1670, 1625, 1496, 1455, 1377, 1307, 1272, 1232; ${ }^{1} \mathrm{H}$ NMR $\left(\mathrm{CDCl}_{3}\right)$ trans isomer: $\delta 0.92(\mathrm{~d}, 3 \mathrm{H}, J$ $=6.8 \mathrm{~Hz}), 1.73(\mathrm{~s}, 3 \mathrm{H}), 2.80(\mathrm{dq}, 1 \mathrm{H}, J=13,6.6 \mathrm{~Hz}), 4.91(\mathrm{~d}, 1 \mathrm{H}, J=13 \mathrm{~Hz}), 7.31(\mathrm{~s}$, 1H), 7.3 - 7.5 (m, 5H); cis isomer: $\delta 0.90(\mathrm{~d}, 3 \mathrm{H}, J=7.6 \mathrm{~Hz}), 1.74(\mathrm{~s}, 3 \mathrm{H}), 2.60(\mathrm{dq}$, $1 \mathrm{H}, J=7.3,3.2 \mathrm{~Hz}), 5.47(\mathrm{~d}, 1 \mathrm{H}, J=3.2 \mathrm{~Hz}), 7.26(\mathrm{~s}, 1 \mathrm{H}), 7.3-7.5(\mathrm{~m}, 5 \mathrm{H}) ;{ }^{13} \mathrm{C}$ NMR $\left(\mathrm{CDCl}_{3}\right)$; trans isomer: $\delta 10.3,10.7,44.7,86.9,113.1,127.3,128.7,129.0$, 137.5, 158.7, 194.9. cis isomer: $\delta 9.9,10.7,45.7,82.9,112.5,125.4,127.9,128.5$, 136.8, 158.8, 197.7; cis isomer HPLC, Daicel Chiralcel OD, hexane $/{ }^{i} \mathrm{PrOH}=40 / 1$, flow rate $=0.6 \mathrm{~mL} / \mathrm{min}: t_{\mathrm{R}}=22.3 \mathrm{~min}\left(\right.$ minor), $t_{\mathrm{R}}=27.1 \mathrm{~min}$ (major); trans isomer HPLC, Daicel Chiralcel OD, hexane $/ \mathrm{PrOH}=19 / 1$, flow rate $=0.3 \mathrm{~mL} / \mathrm{min}: t_{\mathrm{R}}=$ $29.3 \min \left(\right.$ minor), $t_{\mathrm{R}}=31.2 \mathrm{~min}$ (major); trans isomer: HRMS (m/z) calcd. for $\mathrm{C}_{13} \mathrm{H}_{14} \mathrm{O}_{2}\left(\mathrm{M}^{+}\right):$202.0994; found: 202.0993.

3,5-Dimethyl-2-(p-chlorophenyl)-2,3-dihydro-4H-pyran-4-one: trans isomer: $[\alpha]_{\mathrm{D}}{ }^{26}=-59.5\left(\mathrm{c} 1.03, \mathrm{CDCl}_{3}\right)(97 \% \mathrm{ee}) ; \mathrm{IR}\left[\mathrm{cm}^{-1}\right] 2930,1668,1630,1455,1384$ 1306, 1232; ${ }^{1} \mathrm{H} \mathrm{NMR}\left(\mathrm{CDCl}_{3}\right)$ trans isomer: $\delta 0.92(\mathrm{~d}, 3 \mathrm{H}, J=6.8 \mathrm{~Hz}), 1.72(\mathrm{~s}, 3 \mathrm{H})$, $2.74(\mathrm{dq}, 1 \mathrm{H}, J=14,6.8 \mathrm{~Hz}), 4.89(\mathrm{~d}, 1 \mathrm{H}, J=13 \mathrm{~Hz}), 7.30(\mathrm{~s}, 1 \mathrm{H}), 7.3-7.4(\mathrm{~m}, 4 \mathrm{H})$; cis isomer: $\delta 0.88(\mathrm{~d}, 3 \mathrm{H}, J=7.3 \mathrm{~Hz}), 1.74(\mathrm{~s}, 3 \mathrm{H}), 2.56(\mathrm{dq}, 1 \mathrm{H}, J=7.3,3.2 \mathrm{~Hz})$, $5.44(\mathrm{~d}, 1 \mathrm{H}, J=2.9 \mathrm{~Hz}), 7.2-7.4(\mathrm{~m} .5 \mathrm{H}) ;{ }^{13} \mathrm{C} \mathrm{NMR}\left(\mathrm{CDCl}_{3}\right)$; trans isomer: $\delta 10.3$, $10.7,44.7,86.0,113.3,128.7,128.9,134.8,136.1,158.5,194.5$; cis isomer: $\delta 9.9$, 10.6, 45.5, 82.2, 112.7, 126.8, 128.7, 133.8, 135.3, 158.5, 197.3; cis isomer HPLC, Daicel Chiralcel OD, hexane $/{ }^{i} \mathrm{PrOH}=40 / 1$, flow rate $=0.6 \mathrm{~mL} / \mathrm{min}: t \mathrm{R}=14.5 \mathrm{~min}$ (minor), $t \mathrm{R}=27.8 \mathrm{~min}$ (major); trans isomer HPLC, Daicel Chiralcel OD, hexane $/ i \mathrm{PrOH}=19 / 1$, flow rate $=0.3 \mathrm{~mL} / \mathrm{min}: t \mathrm{R}=23.9 \mathrm{~min}($ minor $), t \mathrm{R}=24.9 \mathrm{~min}$ (major); trans isomer: HRMS (m/z) calcd. for $\mathrm{C}_{13} \mathrm{H}_{13} \mathrm{ClO}_{2}\left(\mathrm{M}^{+}\right)$: 236.0604; found: 236.0590; trans isomer: Elemental analysis for $\mathrm{C}_{13} \mathrm{H}_{13} \mathrm{ClO}_{2}$, calcd.: C 65.97, H 5.54; found: C 65.75, H 5.75. 
3,5-Dimethyl-2-(p-tolyl)-2,3-dihydro-4H-pyran-4-one: trans isomer: $[\alpha]_{\mathrm{D}}{ }^{23}=$ $-48.6\left(\mathrm{c} \mathrm{1.48}, \mathrm{CHCl}_{3}\right)\left(90 \%\right.$ ee); IR $\left[\mathrm{cm}^{-1}\right]$ 3445, 2974, 2324, 1671, 1626, 1517, $1456,1376,1305,1270,1233 ;{ }^{1} \mathrm{H}$ NMR $\left(\mathrm{CDCl}_{3}\right)$ trans isomer: $\delta 0.92(\mathrm{~d}, 3 \mathrm{H}, J=6.9$ Hz), 1.72 (s, 3H), 2.73 (s, 3H), 2.80 (dq, 1H, J=13, $6.8 \mathrm{~Hz}), 4.87$ (d, 1H, J=13 Hz), 7.2 - $7.3(\mathrm{~m}, 5 \mathrm{H})$; cis isomer: $\delta 0.90(\mathrm{~d}, 3 \mathrm{H}, J=7.3 \mathrm{~Hz}), 1.73(\mathrm{~s}, 3 \mathrm{H}), 2.37(\mathrm{~s}, 3 \mathrm{H})$, $2.57(\mathrm{dq}, 1 \mathrm{H}, J=7.3,3.3 \mathrm{~Hz}), 5.43(\mathrm{~d}, 1 \mathrm{H}, J=3.1 \mathrm{~Hz}), 7.2-7.3(\mathrm{~m}, 4 \mathrm{H}), 7.37$ (s, $1 \mathrm{H}) ;{ }^{13} \mathrm{C} \mathrm{NMR}\left(\mathrm{CDCl}_{3}\right)$; trans isomer: $\delta 10.4,10.7,21.2,44.6,86.8,113.0,127.3$, 129.3, 134.6, 138.9, 158.8, 195.1. cis isomer: $\delta 9.9,10.7,21.1,45.7,83.0,112.4$, 125.4, 129.1, 133.8, 137.7, 158.9, 197.9; cis isomer HPLC, Daicel Chiralcel OD, hexane $/ i \operatorname{PrOH}=40 / 1$, flow rate $=0.6 \mathrm{~mL} / \mathrm{min}: t_{\mathrm{R}}=15.7 \mathrm{~min}($ major $), t_{\mathrm{R}}=18.1 \mathrm{~min}$ (minor); trans isomer HPLC, Daicel Chiralcel OD, hexane $/ \mathrm{PrOH}=19 / 1$, flow rate $=$ $0.3 \mathrm{~mL} / \mathrm{min}: t_{\mathrm{R}}=22.9 \mathrm{~min}$ (major), $t_{\mathrm{R}}=26.1 \mathrm{~min}$ (minor); trans isomer: HRMS (m/z) calcd. for $\mathrm{C}_{14} \mathrm{H}_{16} \mathrm{O}_{2}\left(\mathrm{M}^{+}\right)$: 216.1150; found: 216.1128 .

3,5-Dimethyl-2-styryl-2,3-dihydro-4H-pyran-4-one: trans isomer: $[\alpha]_{\mathrm{D}}{ }^{19}=$ -28.9 (c 0.3, $\left.\mathrm{CHCl}_{3}\right)(78 \%$ ee); IR [cm-1] 3034, 2816, 2741, 2355, 1675, 1627, 1497, 1450, 1382, 1302; ${ }^{1} \mathrm{H}$ NMR $\left(\mathrm{CDCl}_{3}\right)$ trans isomer: $\delta 1.13(\mathrm{~d}, 3 \mathrm{H}, J=7.0 \mathrm{~Hz}), 1.69(\mathrm{~s}$, 3H), 2.57 (dd, 1H, $J=12,7.0 \mathrm{~Hz}), 4.57$ (dd, 1H, $J=13,7.7 \mathrm{~Hz}), 6.27$ (dd, 1H, $J=16$, $7.7 \mathrm{~Hz}), 6.71(\mathrm{~d}, 1 \mathrm{H}, J=16 \mathrm{~Hz}), 7.2-7.4(\mathrm{~m}, 6 \mathrm{H})$; cis isomer: $\delta 1.1(\mathrm{~d}, 3 \mathrm{H}, J=7.3$ Hz), 1.70 (s, 3H), $2.58(\mathrm{dd}, 1 \mathrm{H}, J=7.3,3.7 \mathrm{~Hz}), 5.01(\mathrm{dd}, 1 \mathrm{H}, J=6.2,3.7 \mathrm{~Hz}), 6.25$ $(\mathrm{dd}, 1 \mathrm{H}, J=16,6.4 \mathrm{~Hz}), 6.74(\mathrm{~d}, 1 \mathrm{H}, J=16 \mathrm{~Hz}), 7.2-7.4(\mathrm{~m}, 6 \mathrm{H}) ;{ }^{13} \mathrm{C} \mathrm{NMR}$ $\left(\mathrm{CDCl}_{3}\right)$; trans isomer: $\delta 10.70,10.74,43.9,85.1,113.0,125.3,126.7,128.4,128.7$, 135.0, 135.7, 158.4, 194.8. cis isomer: $\delta 10.1,10.6,44.1,82.3,112.4,123.2,126.6$, 128.2, 128.6, 133.7, 135.9, 158.3, 196.9; HPLC, Daicel Chiralcel OD, hexane/ ${ }^{\mathrm{PPOH}}$ $=40 / 1$, flow rate $=1.0 \mathrm{~mL} / \mathrm{min}:$ cis isomer $t_{\mathrm{R}}=12.8 \mathrm{~min}\left(\right.$ minor), $t_{\mathrm{R}}=31.7 \mathrm{~min}$ (major); trans isomer $t_{\mathrm{R}}=18.7 \mathrm{~min}$ (major), $t_{\mathrm{R}}=22.0 \mathrm{~min}$ (minor); trans isomer: HRMS (m/z) calcd. for $\mathrm{C}_{15} \mathrm{H}_{16} \mathrm{O}_{2}\left(\mathrm{M}^{+}\right)$: 228.1150; found: 228.1131 .

3,5-Dimethyl-2-phenethyl-2,3-dihydro-4H-pyran-4-one: trans isomer: $[\alpha]_{\mathrm{D}}^{22}=-121.0\left(\mathrm{c} 1.01, \mathrm{CHCl}_{3}\right)(87 \%$ ee $) ; \mathrm{IR}\left[\mathrm{cm}^{-1}\right] 3441,3026,2927,1670,1626$, $1496,1455,1382,1311,1273,1232 ;{ }^{1} \mathrm{H}$ NMR $\left(\mathrm{CDCl}_{3}\right)$ trans isomer: $\delta 1.16(\mathrm{~d}, 3 \mathrm{H}, J$ 
$=7.1 \mathrm{~Hz}), 1.67(\mathrm{~s}, 3 \mathrm{H}), 2.02(\mathrm{dt}, 2 \mathrm{H}, J=14,7.3 \mathrm{~Hz}), 2.43(\mathrm{dt}, 1 \mathrm{H}, J=12,7 \mathrm{~Hz})$, 2.67-2.93 (m, 2H), $4.01(\mathrm{~m}, 1 \mathrm{H}), 7.2$ - $7.4(\mathrm{~m}, 6 \mathrm{H}) ;{ }^{13} \mathrm{C} \mathrm{NMR}\left(\mathrm{CDCl}_{3}\right)$; trans isomer: $\delta 10.7,30.8,34.3,43.5,82.8,112.7,126.0,128.4,128.5,141.2,158.4,195.3 ;$ HPLC, Daicel Chiralcel OD, hexane $/ \mathrm{PrOH}=40 / 1$, flow rate $=1.0 \mathrm{~mL} / \mathrm{min}:$ cis isomer $t_{\mathrm{R}}=$ $12.8 \min (\operatorname{minor}), t_{\mathrm{R}}=31.7 \min \left(\right.$ major); trans isomer $t_{\mathrm{R}}=18.7$ min (major), $t_{\mathrm{R}}=$ $22.0 \mathrm{~min}$ (minor); trans isomer: $\mathrm{HRMS}(\mathrm{m} / \mathrm{z})$ calcd. for $\mathrm{C}_{15} \mathrm{H}_{18} \mathrm{O}_{2}\left(\mathrm{M}^{+}\right)$: 230.1307; found: 230.1315 .

3,5-Dimethyl-2-pentyl-2,3-dihydro-4H-pyran-4-one: trans isomer: $[\alpha]_{\mathrm{D}}{ }^{22}=$ -109.0 (c 1.00, $\left.\mathrm{CHCl}_{3}\right)(88 \%$ ee); IR [cm-1] 3324, 2929, 2860, 1671, 1628, 1458, 1380, 1312, 1271, 1228; ${ }^{1} \mathrm{H} \mathrm{NMR}\left(\mathrm{CDCl}_{3}\right)$ trans isomer: $\delta 0.90(\mathrm{t}, 3 \mathrm{H}, J=7.1 \mathrm{~Hz})$, $1.11(\mathrm{~d}, 3 \mathrm{H}, J=6.8 \mathrm{~Hz}), 1.3-1.6(\mathrm{~m}, 8 \mathrm{H}), 1.60(\mathrm{~d}, 3 \mathrm{H}, J=1.0 \mathrm{~Hz}), 2.35-2.44(\mathrm{~m}$, $1 \mathrm{H}), 4.01(\mathrm{dt}, 1 \mathrm{H}, J=12,5.6 \mathrm{~Hz}), 7.21(\mathrm{~s}, 1 \mathrm{H}) ;{ }^{13} \mathrm{C} \mathrm{NMR}\left(\mathrm{CDCl}_{3}\right)$; trans isomer: $\delta$ $10.69,10.77,13.9,22.5,24.1,31.6,32.3,43.4,83.8$, , 112.4, 158.6, 195.7; cis isomer HPLC, Daicel Chiralcel OD, hexane $/{ }^{i} \mathrm{PrOH}=1000 / 1$, flow rate $=1.0 \mathrm{~mL} / \mathrm{min}: t_{\mathrm{R}}=$ $13.7 \mathrm{~min}$ (minor), $t_{\mathrm{R}}=17.6 \mathrm{~min}$ (major); trans isomer HPLC, Daicel Chiralcel OB-H, hexane $/ i \mathrm{PrOH}=200 / 1$, flow rate $=0.5 \mathrm{~mL} / \mathrm{min}: t_{\mathrm{R}}=13.0 \mathrm{~min}$ (major), $t_{\mathrm{R}}=14.5 \mathrm{~min}$ (minor); trans isomer: HRMS (m/z) calcd. for $\mathrm{C}_{12} \mathrm{H}_{20} \mathrm{O}_{2}\left(\mathrm{M}^{+}\right)$: 196.1463; found: 196.1464 .

$(2 R, 3 R)-3-m e t h y l-2-((E)-1-p r o p e n y l)-2,3-d i h y d r o-4 H-p y r a n-4-o n e ~(t r a n s-$ 3): trans-3: IR [cm-1] 1677, 1599, 1457, 1409, 1377, 1308, 1285, 1250, 1217. ${ }^{1} \mathrm{H}$ NMR $\left(\mathrm{CDCl}_{3}\right) \delta 1.04(\mathrm{~d}, 3 \mathrm{H}, J=7.1 \mathrm{~Hz}), 1.76(\mathrm{dd}, 3 \mathrm{H}, \mathrm{J}=6.6,1.7 \mathrm{~Hz}), 2.46(\mathrm{dq}, 1 \mathrm{H}$, $J=12,7.1 \mathrm{~Hz}), 4.39(\mathrm{dd}, 1 \mathrm{H}, J=12,8.3), 5.36(\mathrm{~d}, 1 \mathrm{H}, J=5.8 \mathrm{~Hz}), 5.57(\mathrm{ddq}, 1 \mathrm{H}, J=$ 15, 8.3, $1.7 \mathrm{~Hz}), 5.86(\mathrm{ddq}, 1 \mathrm{H}, J=15,6.6,0.7 \mathrm{~Hz}), 7.30$ (d, $1 \mathrm{H}, J=6.1 \mathrm{~Hz}) .{ }^{13} \mathrm{C}$ $\mathrm{NMR}\left(\mathrm{CDCl}_{3}\right) \delta 10.6,17.7,43.9,85.5,106.2,127.4,132.9,162.2,194.7$. MS [m/z] $152\left(\mathrm{M}^{+}\right),[\alpha]_{\mathrm{D}}^{22}=+83.1\left(\mathrm{c} 1.35, \mathrm{CHCl}_{3}, 87 \%\right.$ ee). . HRMS (m/z) calcd. for $\mathrm{C}_{9} \mathrm{H}_{12} \mathrm{O}_{2}\left(\mathrm{M}^{+}\right):$152.0837; found: 152.0831 . The ratio of trans- and cis- isomers was determined by ${ }^{1} \mathrm{H}$ NMR analysis, and the optical purity was determined by HPLC analysis (Daicel Chiralcel AS, hexane $/{ }^{i} \mathrm{PrOH}=100 / 1$, flow rate $=1.0 \mathrm{~mL} / \mathrm{min}$ : trans isomer $t_{\mathrm{R}}=23.1 \mathrm{~min}$ (major), $t_{\mathrm{R}}=19.5 \mathrm{~min}$ (minor); cis isomer $t_{\mathrm{R}}=30.0 \mathrm{~min}$ 
(major), $t_{\mathrm{R}}=48.0 \mathrm{~min}$ (minor)). Then, trans $\mathbf{- 3}$ was separated by column chromatography (silica gel, P.E.-diethyl ether=9:1) and used in the next reaction.

$(2 R, 3 S, 4 S)-4-H y d r o x y-3-m e t h y l-2-((E)-1-p r o p e n y l)-2,3-d i h y d r o-4 H-$ pyrane (4): m.p. $88^{\circ} \mathrm{C}$. IR [cm-1] (KBr) 3246, 1651, 1457, 1378, 1357, 1335, 1312, 1282, 1268, 1233. ${ }^{1} \mathrm{H}$ NMR $\left(\mathrm{CDCl}_{3}\right) \delta 0.98(\mathrm{~d}, 3 \mathrm{H}, J=6.8 \mathrm{~Hz}), 1.38(\mathrm{~d}, 1 \mathrm{H}, J=8.0$ $\mathrm{Hz}), 1.65$ (m, 1H), 1.75 (dd, 3H, J= 6.6, $1.7 \mathrm{~Hz}), 3.88$ (m, 1H), $4.01(\mathrm{~m}, 1 \mathrm{H}), 4.77$ $(\mathrm{dd}, 1 \mathrm{H}, J=6.1,2.2 \mathrm{~Hz}), 5.49(\mathrm{ddq}, 1 \mathrm{H}, J=15,8.0,1.7 \mathrm{~Hz}), 5.79(\mathrm{dq}, 1 \mathrm{H}, J=15,6.4$ $\mathrm{Hz}), 6.39(\mathrm{dd}, 1 \mathrm{H}, J=6.3,1.2 \mathrm{~Hz}) .{ }^{13} \mathrm{C} \mathrm{NMR}\left(\mathrm{CDCl}_{3}\right) \delta 14.9,17.8,40.7,69.1,81.0$, 104.8, 128.9, 130.9, 144.7. MS [m/z] $154\left(\mathrm{M}^{+}\right),[\alpha]_{\mathrm{D}}^{22}=-88.9(\mathrm{c} 0.65, \mathrm{THF},>99 \%$ ee after recrystallization). HRMS (m/z) calcd. for $\mathrm{C}_{9} \mathrm{H}_{14} \mathrm{O}_{2}\left(\mathrm{M}^{+}\right)$: 154.0994; found:154.0997. The enantiomeric excess of the product was determined by HPLC analysis (Daicel Chiralpak AD, hexane $/{ }^{i} \mathrm{PrOH}=1000 / 1$, flow rate $=1.0 \mathrm{~mL} / \mathrm{min}: t_{\mathrm{R}}=$ $20.5 \min \left(\right.$ major), $t_{\mathrm{R}}=15.5 \mathrm{~min}$ (minor)) after benzoylation using benzoyl chloride and pyridine, DMAP.

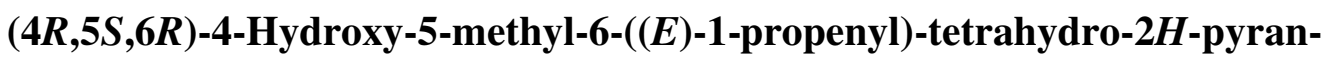
2-one ((+)-Prelactone C, 6): IR [cm-1] (neat) 3434, 1730, 1454, 1376, $1244 .{ }^{1} \mathrm{H}$ $\operatorname{NMR}\left(\mathrm{CDCl}_{3}\right) \delta 1.03(\mathrm{~d}, 3 \mathrm{H}, J=6.7 \mathrm{~Hz}), 1.65(\mathrm{~m}, 1 \mathrm{H}), 1.75(\mathrm{dd}, 3 \mathrm{H}, J=6.8,1.6 \mathrm{~Hz})$, $2.49(\mathrm{dd}, 1 \mathrm{H}, J=17,8.6 \mathrm{~Hz}), 2.57$ (br, 1H), 2.91 (dd, 1H, $J=17,5.8 \mathrm{~Hz}), 3.77$ (m, 1H), 4.19 (dd, 1H, $J=10.8 .6 \mathrm{~Hz}), 5.43(\mathrm{ddq}, 1 \mathrm{H}, J=15,8.2,1.9 \mathrm{~Hz}), 5.80$ (dq, $1 \mathrm{H}, J=$ 15, 6.7 Hz). ${ }^{13} \mathrm{C} \mathrm{NMR}\left(\mathrm{CDCl}_{3}\right) \delta$ 13.7, 17.6, 39.1, 41.5, 69.5, 84.1, 127.6, 132.4, 170.4. $\mathrm{MS}[\mathrm{m} / \mathrm{z}] 170\left(\mathrm{M}^{+}\right), 152\left(\left(\mathrm{M}-\mathrm{H}_{2} \mathrm{O}\right)^{+}\right) .[\alpha]_{\mathrm{D}^{24}}=+65.4(\mathrm{c} 0.71, \mathrm{MeOH})$. lit. $)^{3}$ $[\alpha]_{\mathrm{D}^{20}}=+57.6(\mathrm{c} 0.5, \mathrm{MeOH})$. HRMS $(\mathrm{m} / \mathrm{z})$ calcd. for $\mathrm{C}_{9} \mathrm{H}_{14} \mathrm{O}_{3}\left(\mathrm{M}^{+}\right)$: 170.0943; found: 170.0972 .

\section{References}

(1) Danishefsky's dienes employed were prepared according to the procedures reported in the literatures. (a) Danishefsky, S.; Kitahara, T. J. Am. Chem. Soc. 1974, 96, 7807. (b) Bednarski, M.; Maring, C.; Danishefsky, S. Tetrahedron Lett. 1983, 24, 3451. (c) Myles, D. C.; Bigham, M. H. Organic Synthesis Vol. 
70, Meyers, A. I. Eds., 231. (d) Burger, M. T.; Still. W. C. J. Org. Chem. 1996, 61,775 .

(2) Kezuka, S.; Mita, T.; Ohtsuki, N.; Ikeno, T.; Yamada, T. Bull. Chem. Soc. Jpn., 2001, 74, 1333.

(3) Bindseil, K. U.; Zeeck, A. Helv. Chim. Acta 1993, 76, 150. 